\title{
Stents and surgical interventions in the palliation of gastric outlet obstruction: a systematic review
}

Authors

Institutions
Mauricio Kazuyoshi Minata' ${ }^{1}$, Wanderley Marques Bernardo², Rodrigo Silva de Paula Rocha', Flavio Hiroshi Ananias Morita ${ }^{1}$, Julio Cesar Martins Aquino ${ }^{1}$, Spencer Cheng ${ }^{1}$, Bruno Zilberstein ${ }^{3}$, Paulo Sakai', Eduardo Guimarães Hourneaux de Moura ${ }^{1}$

${ }^{1}$ University of São Paulo Medical School, Gastrointestinal Endoscopy Unit, Gastroenterology Department, São Paulo, Brazil 2 University of São Paulo Medical School, Gastroenterology Department, São Paulo, Brazil

${ }^{3}$ University of São Paulo Medical School, Digestive Surgery, Gastroenterology Department, São Paulo, Brazil submitted 15. April 2016 accepted after revision 29. July 2016

\section{Bibliography}

Dol http://dx.doi.org/

10.1055/s-0042-115935

Published online: 28.10.2016

Endoscopy International Open 2016; 04: E1158-E1170

(c) Georg Thieme Verlag KG

Stuttgart · New York

E-ISSN 2196-9736

\section{Corresponding author} Mauricio Kazuyoshi Minata University of São Paulo Medical School

Gastrointestinal Endoscopy Unit Gastroenterology Department Avenida Dr. Enéas de Carvalho Aguiar, 155

$6^{\circ}$ andar São Paulo SP

05013001 Brazil

Fax: +55112661-0000

mauriciominata@hotmail.com
Background and study aims: Palliative treatment of gastric outlet obstruction can be done with surgical or endoscopic techniques. This systematic review aims to compare surgery and covered and uncovered stent treatments for gastric outlet obstruction (GOO).

Patients and methods: Randomized clinical trials were identified in MEDLINE, Embase, Cochrane, LILACs, BVS, SCOPUS and CINAHL databases. Comparison of covered and uncovered stents included: technical success, clinical success, complications, obstruction, migration, bleeding, perforation, stent fracture and reintervention. The outcomes used to compare surgery and stents were technical success, complications, and reintervention. Patency rate could not be included because of lack of uniformity of the extracted data.

Results: Eight studies were selected, 3 comparing surgery and stents and 5 comparing covered and uncovered stents.The meta-analysis of surgical

\section{Introduction}

$\nabla$

Advanced stage malignancy in the gastrointestinal tract may result in gastric outlet obstruction (GOO), which is characterized by symptoms like abdominal pain, weight loss, nausea and vomiting [1]. Considering that many cases of GOO are unfit for surgical resection, palliative treatment is indicated in order to provide better quality of life [2]. Palliation can be done with surgical or endoscopic stent treatment [3-7].

Surgical gastrojejunostomy is considered a more aggressive choice by some authors, with reports of considerable morbidity and mortality $[8,9]$. Endoscopic treatment with self-expandable metallic stents (SEMS) is a valid method to palliate malignant gastric outlet obstruction [10]. This method is associated with rapid relief of symptoms and low complication rates in the short term [11], although, many patients refuse surgical intervention [12]. However, endoscopic stents, and endoscopic stent treatment showed no difference in the technical success and overall number of complications. Stents had higher reintervention rates than surgery (RD: $0.26,95 \% \mathrm{CI}$ $[0.05,0.47]$, NNH: 4). There is no significant difference in technical success, clinical success, complications, stent fractures, perforation, bleeding and the need for reintervention in the analyses of covered and uncovered stents. There is a higher migration rate in the covered stent therapy compared to uncovered self-expanding metallic stents (SEMS) in the palliation of malignant GOO (RD: 0.09, 95\% CI [0.04, 0.14], NNH: 11). Nevertheless, covered stents had lower obstruction rates (RD: $-0.21,95 \%$ CI [-0.27, -0.15$],$ NNT: 5 ).

Conclusions: In the palliation of malignant GOO, covered SEMS had higher migration and lower obstruction rates when compared with uncovered stents. Surgery is associated with lower reintervention rates than stents.

despite the lower initial morbidity, demonstrated complications like obstruction, migration, bleeding and stent fractures [13].

The perfect treatment should combine high technical and clinical success, with low complication rates and low need for reintervention. The rationale is that endoscopic stenting is a less invasive option for treatment than surgery, but one key point is the patency rate and need for reintervention.

Another point to highlight is the choice of different kinds of stents. Uncovered SEMS presents higher obstruction rates because of tumor ingrowth through the mesh. Covered SEMS present lower obstruction, but higher migration rates [14].

With the development of new technologies and new randomized trials, we expect changes in the management of gastric outlet obstruction, a fact that demands analysis of which kind of therapy is better and if there is a difference between cov- 
ered and uncovered stents [15-17]. The published systematic reviews with meta-analyses about this subject does not include those new randomized clinical trials and considered analysis with non-randomized trials together [18-24]. Our systematic review aims to compare the outcomes of randomized studies of surgical versus endoscopic stenting and covered versus uncovered stents.

\section{Methods}

$\nabla$

Systematic review conducted in accordance with the PRISMA (preferred reporting items for systematic reviews and meta-analyses) recommendations and registered on the PROSPERO international database (CRD42016032939) [25].

\section{Eligibility criteria}

Inclusion criteria: only complete published randomized clinical trials (RCT) comparing palliative treatment of malignant GOO with surgery and covered and uncovered stent treatment. No restrictions for language or year of publication were applied. Exclusion criteria: abstracts, studies including patients with prior stent or surgical treatment for GOO.

Outcome measures for surgical and stent comparison: number of patients with complications, technical success and reintervention.

Outcome measures for covered and uncovered stents: technical success, clinical success, complications, migration, obstruction, bleeding, perforation, fracture and reintervention.

\section{Search and information sources}

Studies were identified by searching electronic databases (MEDLINE, Embase, Cochrane, Scopus, LILACS, BVS and CINAHL). The grey literature search included chapters of endoscopy and gastroenterology books, theses and references in the selected articles and in published systematic reviews. Last search was run on October 31, 2015.

Search terms included in the MEDLINE were (gastric outlet obstruction OR gastric outlet obstructions OR duodenal obstruction) AND (endoscopy OR endoscopic OR endoscopic surgical procedure OR endoscopic surgical procedures OR stent OR stents) AND random*. In the other databases, the same strategy was used with a few modifications. Full search strategy is available in Appendix 1.

\section{Study selection and data collection process}

Two reviewers performed eligibility assessment and selection of screened records independently in an unblinded, standardized manner. Disagreements were resolved by consensus. In case of duplicated publications, the most complete and recent was selected. The same authors extracted data from selected studies using a standardized form (Supplementary Information Sheet). Disagreements were resolved by consensus.

\section{Data items}

Information was extracted from each trial on: (1) characteristics of the trials participants and trial's inclusion and exclusion criteria; (2) type of intervention and control groups (results divided into groups, one with surgical and stent comparison, another with covered and uncovered stents); (3) type of outcome measures. Technical success was defined as an adequate and accurate stent positioning in the stricture area or adequate gastrojejunost- omy. Clinical success was defined as the clinical relief of obstruction symptoms after intervention. Complications were considered as reported or the sum of all adverse events informed. Perforation and fracture were accounted as reported and were considered absent if not specified. Stents were classified as covered and uncovered. Covered stents included in the studies are not fully covered.

\section{Risk of bias in individual studies}

Two reviewers analyzed together the quality of the studies with the Jadad scale, in order to certify the adequacy of randomization, concealment of allocation, blinding and follow up report. This score varies from 0 to 5 , with scores below 3 indicating poor methodological quality.

\section{Summary measures and planned methods of analysis}

All statistical calculations were carried out using the computer software programs OpenEpi and RevMan five version 5.3.The meta-analyses were performed with Review Manager 5.3 software (RevMan), which was obtained from Cochrane Informatics \& Knowledge Management Department (http://tech.cochrane. org/revman). Dichotomous data was analyzed by computing Risk differences (RD) with a fixed effect model, Mantel-Haenszel test and intention-to-treat analysis (ITT).

We calculated 95\% confidence intervals (95\% CI), number necessary to treat (NNT) or to harm (NNH) for each outcome and study. Graphical analysis with funnel plot and forest plot were generated. Inconsistency (heterogeneity) was calculated using the Chi-square test $\left(\mathrm{Chi}^{2}\right)$ and the Higgins method $\left(\mathrm{I}^{2}\right)$. The advantages of the Higgins method are that it does not depend on the number of studies and it is accompanied by an uncertainty interval. A cut-off point of $\mathrm{I}^{2}<50 \%$ was established as acceptable.

\section{Risk of bias across studies}

A graphical method was used (forest plots) to evaluate the relation between sample size and effect size for each outcome. Funnel plots were used for evaluate the risk of publication bias across the studies outcomes. The graphical method analysis involved a plot of the trials mean differences and search for asymmetry. Quantification of heterogeneity is another component of the investigation of variability across studies. Considering the clinical implications of the observed degree of inconsistency across studies, the cutoff value of $50 \%$ was considered adequate for this meta-analysis. If the heterogeneity of the results of a meta-analysis $\left(\mathrm{I}^{2}\right)$ was over $50 \%$, a sensitivity analysis was executed, excluding the reports located outside the funnel (outliers) and then performed another meta-analysis without the given report. In case of persistent high heterogeneity after this process or if we could not detect outliers, true heterogeneity was presumed and a random model was assumed.

We acknowledge that other factors could produce asymmetry in funnel plots leading to a high heterogeneity (true study heterogeneity), such as differences in the population studied, differences in trial quality or even different techniques studied under the same endoscopic modality (different stents).

\section{Additional analyses}

For the comparison of stents, a subgroup analysis was generated with the trials that included only patients with gastric cancer. 


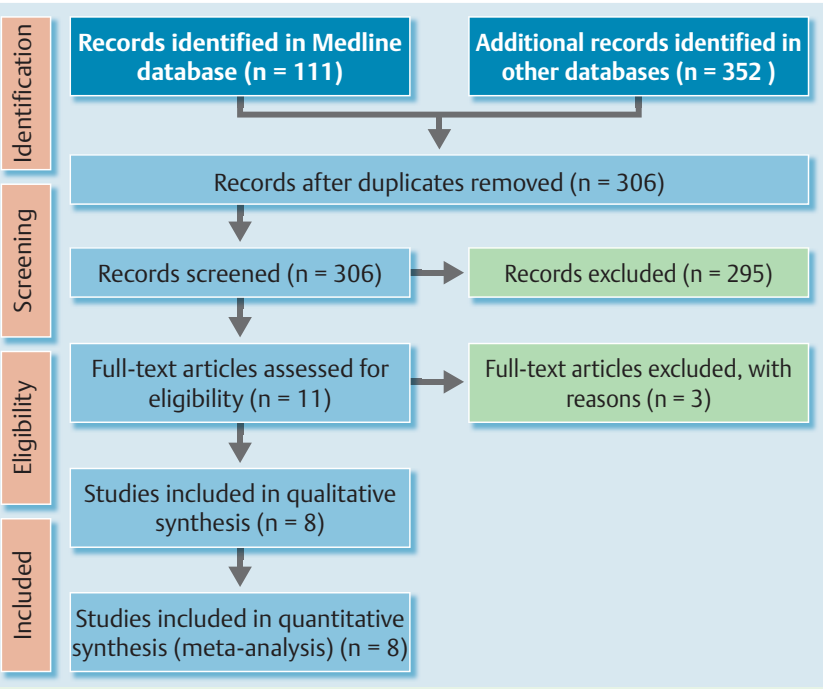

Fig. 1 Flow diagram of study selection

\section{Results}

The literature search resulted in 111 records in MEDLINE and 352 in the other databases. Six trials were included in the initial selection of articles on surgery and stents [11,12,26-29]. One was excluded because it used randomized and observational samples together [29]. Two studies were duplicated $[11,28]$. The most recent and complete were included for meta-analysis. Finally, 5 clinical randomized trials were selected for covered and uncovered SEMS, including 443 patients [15-17,30,31]. Three studies were considered for comparison between surgery and stents, with a total of 84 patients $[12,26,27]$. In the grey literature search, there was no new complementation. The study selection process is illustrated in $\bullet$ Fig. 1. Study characteristics, risk of bias and individual results are represented on $\bullet$ Table $1, \bullet$ Table 2 , Table 3.

\section{Surgical treatment versus stents}

Technical success

For both groups there is a high success rate, with no significant difference (RD: - 0.05, 95\% CI [-0.16, 0.07], I²: 0\%) ( Fig. 2).

\section{Complications}

For this outcome we noticed high heterogeneity $\left(\mathrm{I}^{2}: 85 \%\right)$. There is one outlier trial [26]. After excluding this study, there is no significant difference (RD: $0.07,95 \%$ CI $[-0.17,0.31], \mathrm{I}^{2}: 0 \%$ ) ( $\bullet$ Fig.3).

\section{Reintervention}

Results are favorable to the surgery group, with less need for reinterventions (RD: $0.26,95 \% \mathrm{CI}[0.05,0.47], \mathrm{I}^{2}$ : $0 \%, \mathrm{NNH}: 4$ ) (๑ Fig.4).

\section{Covered versus uncovered stents}

Technical and Clinical Success

High technical success was noticed, with no significant difference between covered and uncovered SEMS (RD: 0.00, 95\% CI [-0.04, $\left.0.04], I^{2}: 0 \%\right)$. no significant difference was attributed for clinical success (RD: $0.02,95 \%$ CI [-0.03, 0.07], I ${ }^{2}$ : 0\%). Both had high success rates and low heterogeneity. ( $\bullet$ Fig. 5 and $\bullet$ Fig. 6$)$.

Table 1 Characteristics of selected studies.

\begin{tabular}{|c|c|c|c|c|c|c|}
\hline Study & Population (N) & Intervention (N) & Comparison (N) & Outcomes & Follow up & Centers \\
\hline \multicolumn{7}{|c|}{ Covered and uncovered stents } \\
\hline Shi 2014 & 65 & $\begin{array}{l}\text { Tailored covered } \\
\text { stents Micro-Tech } \\
\text { (33) }\end{array}$ & $\begin{array}{l}\text { Uncovered stents MTN- } \\
\text { CG-S-20/100 Micro-Tech } \\
\text { (32) }\end{array}$ & $\begin{array}{l}\text { Technical success, clinical suc- } \\
\text { cess, complications, obstruction, } \\
\text { migration, bleeding, perforation, } \\
\text { fracture, reintervention }\end{array}$ & Until death & $\begin{array}{l}\text { Multicenter } \\
\text { ( } 3 \text { centers) }\end{array}$ \\
\hline Lim 2014 & 134 & $\begin{array}{l}\text { Covered double } \\
\text { layered - Niti-S } \\
\text { ComVi pyloric } \\
\text { Stent (66) }\end{array}$ & $\begin{array}{l}\text { Uncovered Niti-S pyloric/ } \\
\text { duodenal D-type stent } \\
\text { (68) }\end{array}$ & $\begin{array}{l}\text { Technical success, clinical suc- } \\
\text { cess, complications, obstruction, } \\
\text { migration, bleeding, perforation, } \\
\text { fracture, reintervention }\end{array}$ & Unclear & $\begin{array}{l}\text { Multicenter } \\
\text { (4 centers) }\end{array}$ \\
\hline Kim 2010 & 80 & $\begin{array}{l}\text { Covered Niti-S py- } \\
\text { loric Stent and Niti- } \\
\text { S ComVi pyloric } \\
\text { Stents (40) }\end{array}$ & $\begin{array}{l}\text { Uncovered enteral } \\
\text { Wallstents and Wallflex } \\
\text { duodenal stents }(40)\end{array}$ & $\begin{array}{l}\text { Technical success, clinical suc- } \\
\text { cess, complications, obstruction, } \\
\text { migration, bleeding, perforation, } \\
\text { fracture }\end{array}$ & Unclear & $\begin{array}{l}\text { Single- } \\
\text { center }\end{array}$ \\
\hline Maetani 2014 & 62 & $\begin{array}{l}\text { Covered triple } \\
\text { layered ComVi } \\
\text { stent (31) }\end{array}$ & $\begin{array}{l}\text { Uncovered Niti-S stent } \\
\text { (31) }\end{array}$ & $\begin{array}{l}\text { Technical success, clinical suc- } \\
\text { cess, complications, obstruction, } \\
\text { migration, bleeding, perforation, } \\
\text { fracture }\end{array}$ & Until death & $\begin{array}{l}\text { Multicenter } \\
\text { ( } 2 \text { centers) }\end{array}$ \\
\hline Lee 2015 & 102 & $\begin{array}{l}\text { Bonastent WAVE- } \\
\text { covered SEMS }(51)\end{array}$ & $\begin{array}{l}\text { Bonastent uncovered } \\
\text { SEMS (51) }\end{array}$ & $\begin{array}{l}\text { Technical success, clinical suc- } \\
\text { cess, complications, obstruction, } \\
\text { migration, bleeding, perforation, } \\
\text { fracture, reintervention }\end{array}$ & $\begin{array}{l}\text { Until death or } \\
\text { censoring date } \\
\text { of } 30 \text { november } \\
2014\end{array}$ & $\begin{array}{l}\text { Multicenter } \\
\text { (5 centers) }\end{array}$ \\
\hline \multicolumn{7}{|c|}{ Surgery and stents } \\
\hline Mehta, 2006 & 27 & $\begin{array}{l}\text { Uncovered Enteral } \\
\text { Wallstent (13) }\end{array}$ & Laparoscopic GJJ (14) & Technical success, complications & not clear & $\begin{array}{l}\text { Not de- } \\
\text { scribed }\end{array}$ \\
\hline $\begin{array}{l}\text { Jeurnink, } \\
2010\end{array}$ & 39 & $\begin{array}{l}\text { Uncovered Enteral } \\
\text { Wallstent (21) }\end{array}$ & $\begin{array}{l}\text { Open or laparoscopic GJJ } \\
\text { (18) }\end{array}$ & $\begin{array}{l}\text { Technical success, complica- } \\
\text { tions, reintervention }\end{array}$ & Until death & $\begin{array}{l}\text { Multicenter } \\
\text { ( } 21 \text { centers) }\end{array}$ \\
\hline Fiori, 2013 & 18 & $\begin{array}{l}\text { Ultraflex Covered } \\
\text { Stent (9) }\end{array}$ & Open GJJ (9) & $\begin{array}{l}\text { Technical success, complica- } \\
\text { tions, reintervention }\end{array}$ & Until death & $\begin{array}{l}\text { Single- } \\
\text { center }\end{array}$ \\
\hline
\end{tabular}


Table 2 Risk of bias and Jadad.

\begin{tabular}{|c|c|c|c|c|c|c|c|c|c|c|c|}
\hline Study & $\begin{array}{l}\text { Popula- } \\
\text { tion (N) }\end{array}$ & Question & $\begin{array}{l}\text { Randomi- } \\
\text { zation }\end{array}$ & $\begin{array}{l}\text { Alloca- } \\
\text { tion }\end{array}$ & Blinding & Losses & Prognostic & ITT & $\begin{array}{l}\text { Sample } \\
\text { size }\end{array}$ & $\begin{array}{l}\text { Selection } \\
\text { bias }\end{array}$ & JADAD \\
\hline \multicolumn{12}{|c|}{ Covered and uncovered stent studies } \\
\hline Shi 2014 & $\begin{array}{l}65(33 \\
\text { covered } \\
\text { and } 32 \\
\text { uncov- } \\
\text { ered) }\end{array}$ & $\begin{array}{l}\text { To compare the effi- } \\
\text { cacy and safety of } \\
\text { "outlet-shape" tai- } \\
\text { lored stents with } \\
\text { standard stents for } \\
\text { the management of } \\
\text { distal gastric cancer } \\
\text { causing GO with } \\
\text { varying gastric cav- } \\
\text { ity shapes and sizes }\end{array}$ & $\begin{array}{l}\text { Table of } \\
\text { random } \\
\text { numbers }\end{array}$ & $\begin{array}{l}\text { Not de- } \\
\text { scribed }\end{array}$ & $\begin{array}{l}\text { Not de- } \\
\text { scribed }\end{array}$ & $\begin{array}{l}1 \text { covered } \\
(3,03 \%) \\
\text { and } 1 \text { un- } \\
\text { covered } \\
(3,12 \%)\end{array}$ & $\begin{array}{l}\text { Homoge- } \\
\text { neous }\end{array}$ & No & Yes & $\begin{array}{l}\text { Only gas- } \\
\text { tric can- } \\
\text { cer }\end{array}$ & 3 \\
\hline $\operatorname{Lim} 2014$ & $\begin{array}{l}134(66 \\
\text { covered } \\
\text { and } 68 \\
\text { uncov- } \\
\text { ered) }\end{array}$ & $\begin{array}{l}\text { To evaluate out- } \\
\text { comes after place- } \\
\text { ment of conform- } \\
\text { able covered and } \\
\text { uncovered self-ex- } \\
\text { pandable metallic } \\
\text { stents for palliation } \\
\text { of malignant GOO }\end{array}$ & $\begin{array}{l}\text { Computer- } \\
\text { generated } \\
\text { list }\end{array}$ & $\begin{array}{l}\text { Not de- } \\
\text { scribed }\end{array}$ & Patient & $\begin{array}{l}7 \text { covered } \\
(10,6 \%) \\
\text { and } 7 \text { un- } \\
\text { covered } \\
(10,29 \%)\end{array}$ & $\begin{array}{l}\text { Homoge- } \\
\text { neous }\end{array}$ & No & Yes & No & 3 \\
\hline Kim 2010 & $\begin{array}{l}80 \text { ( } 40 \\
\text { covered } \\
\text { and } 40 \\
\text { uncov- } \\
\text { ered) }\end{array}$ & $\begin{array}{l}\text { To compare the ef- } \\
\text { fectiveness and side } \\
\text { effects of covered } \\
\text { and uncovered } \\
\text { SEMSs for the pallia- } \\
\text { tion of malignant } \\
\text { pyloric obstruction }\end{array}$ & $\begin{array}{l}\text { Computer- } \\
\text { generated } \\
\text { list }\end{array}$ & $\begin{array}{l}\text { Not de- } \\
\text { scribed }\end{array}$ & $\begin{array}{l}\text { Patient } \\
\text { blinded }\end{array}$ & $\begin{array}{l}2 \text { uncov- } \\
\text { ered (5\%) } \\
\text { and } 5 \\
\text { covered } \\
(12,5 \%)\end{array}$ & $\begin{array}{l}\text { Homoge- } \\
\text { neous }\end{array}$ & No & Yes & $\begin{array}{l}\text { only gas- } \\
\text { tric can- } \\
\text { cer }\end{array}$ & 3 \\
\hline $\begin{array}{l}\text { Maetani } \\
2014\end{array}$ & $\begin{array}{l}62 \text { ( } 31 \\
\text { covered } \\
\text { and } 31 \\
\text { uncov- } \\
\text { ered) }\end{array}$ & $\begin{array}{l}\text { To compare the effi- } \\
\text { cacy and safety of a } \\
\text { triple-layered cov- } \\
\text { ered versus uncov- } \\
\text { ered SEMS }\end{array}$ & $\begin{array}{l}\text { sealed en- } \\
\text { velops }\end{array}$ & $\begin{array}{l}\text { Equal ra- } \\
\text { tio conse- } \\
\text { cutively } \\
\text { by using } \\
\text { sealed en- } \\
\text { velopes }\end{array}$ & $\begin{array}{l}\text { Blinded } \\
\text { in follow } \\
\text { up }\end{array}$ & 0 & $\begin{array}{l}\text { Homoge- } \\
\text { neous }\end{array}$ & Yes & Yes & No & 3 \\
\hline Lee 2015 & $\begin{array}{l}102(51 \\
\text { covered } \\
\text { and } 51 \\
\text { uncov- } \\
\text { ered) }\end{array}$ & $\begin{array}{l}\text { to evaluate and } \\
\text { compare the effica- } \\
\text { cy of WAVE covered } \\
\text { SEMS with uncov- } \\
\text { ered SEMS in GOO }\end{array}$ & $\begin{array}{l}\text { Computer- } \\
\text { generated } \\
\text { list }\end{array}$ & $\begin{array}{l}\text { Equal ra- } \\
\text { tio, Com- } \\
\text { puter- } \\
\text { gener- } \\
\text { ated, with } \\
\text { a block } \\
\text { size of } \\
\text { four }\end{array}$ & Patients & $\begin{array}{l}1 \text { covered } \\
(1,96 \%) \\
\text { and } 4 \text { un- } \\
\text { covered } \\
(7,84 \%)\end{array}$ & $\begin{array}{l}\text { Homoge- } \\
\text { neous }\end{array}$ & Yes & Yes & $\begin{array}{l}\text { Only gas- } \\
\text { tric can- } \\
\text { cer }\end{array}$ & 3 \\
\hline \multicolumn{12}{|c|}{ Surgery and Stent Studies } \\
\hline $\begin{array}{l}\text { Mehta, } \\
2006\end{array}$ & $\begin{array}{l}27(13 \\
\text { stent } \\
\text { and } 14 \\
\text { surgery) }\end{array}$ & $\begin{array}{l}\text { To compare laparo- } \\
\text { scopic gastrojeju- } \\
\text { nostomy with duo- } \\
\text { denal stenting }\end{array}$ & $\begin{array}{l}\text { Computer } \\
\text { generated } \\
\text { lists }\end{array}$ & $\begin{array}{l}\text { Not de- } \\
\text { scribed }\end{array}$ & $\begin{array}{l}\text { Re- } \\
\text { searcher }\end{array}$ & $\begin{array}{l}1 \text { stent } \\
(7,69 \%) \\
\text { and } \\
1 \text { surgical } \\
(7,14 \%)\end{array}$ & $\begin{array}{l}\text { Homoge- } \\
\text { neous }\end{array}$ & Yes & $\begin{array}{l}\text { Not } \\
\text { men- } \\
\text { tioned }\end{array}$ & No & 3 \\
\hline $\begin{array}{l}\text { Jeurnink, } \\
2010\end{array}$ & $\begin{array}{l}39(21 \\
\text { stent } \\
\text { and } 18 \\
\text { surgery) }\end{array}$ & $\begin{array}{l}\text { To compare GJJ and } \\
\text { stent placement }\end{array}$ & $\begin{array}{l}\text { Computer } \\
\text { generated } \\
\text { lists }\end{array}$ & $\begin{array}{l}\text { Not de- } \\
\text { scribed }\end{array}$ & $\begin{array}{l}\text { Not de- } \\
\text { scribed }\end{array}$ & 0 & $\begin{array}{l}\text { Homoge- } \\
\text { neous }\end{array}$ & Yes & $\begin{array}{l}\text { Not } \\
\text { men- } \\
\text { tioned }\end{array}$ & No & 3 \\
\hline $\begin{array}{l}\text { Fiori, } \\
2013\end{array}$ & $\begin{array}{l}18 \text { ( } 9 \\
\text { stent } \\
\text { and } 9 \\
\text { surgery) }\end{array}$ & $\begin{array}{l}\text { To compare the } \\
\text { endoscopic place- } \\
\text { ment of self-ex- } \\
\text { pandable stents } \\
\text { with open surgical } \\
\text { GJJ }\end{array}$ & $\begin{array}{l}\text { Random } \\
\text { number ta- } \\
\text { bles }\end{array}$ & $\begin{array}{l}\text { Not de- } \\
\text { scribed }\end{array}$ & $\begin{array}{l}\text { Pa- } \\
\text { tients/ } \\
\text { re- } \\
\text { searcher }\end{array}$ & 0 & $\begin{array}{l}\text { Homoge- } \\
\text { neous }\end{array}$ & Yes & $\begin{array}{l}\text { Not } \\
\text { men- } \\
\text { tioned }\end{array}$ & $\begin{array}{l}\text { Sympto- } \\
\text { matic pri- } \\
\text { mary ade- } \\
\text { nocarci- } \\
\text { noma of } \\
\text { the antro- } \\
\text { pyloric re- } \\
\text { gion }\end{array}$ & 3 \\
\hline
\end{tabular}


Review

Table 3 Results of individual studies.

\begin{tabular}{|c|c|c|c|c|c|c|c|c|c|c|}
\hline \multicolumn{11}{|c|}{ Covered and uncovered stents } \\
\hline & \multicolumn{2}{|l|}{ Shi 2014} & \multicolumn{2}{|l|}{ Lim 2014} & \multicolumn{2}{|c|}{ Kim 2010} & \multicolumn{2}{|c|}{ Maetani 2014} & \multicolumn{2}{|l|}{ Lee 2015} \\
\hline & Covered & Uncovered & Covered & Uncovered & Covered & Un covered & Covered & Uncovered & Covered & Uncovered \\
\hline Total & 33 & 32 & 66 & 68 & 40 & 40 & 31 & 31 & 51 & 51 \\
\hline Technical success & 32 & 31 & 59 & 61 & 40 & 40 & 31 & 31 & 50 & 49 \\
\hline Clinical success & 31 & 30 & 59 & 60 & 38 & 36 & 27 & 29 & 49 & 46 \\
\hline Complications & 28 & 11 & 13 & 13 & 16 & 19 & 6 & 10 & 7 & 16 \\
\hline Obstruction & 1 & 7 & 4 & 13 & 1 & 16 & 0 & 6 & 3 & 14 \\
\hline Migration & 2 & 0 & 8 & 0 & 10 & 3 & 2 & 1 & 4 & 2 \\
\hline Bleeding & 11 & 2 & 0 & 0 & 0 & 0 & 0 & 1 & 0 & 0 \\
\hline Perforation & 0 & 0 & 0 & 0 & 1 & 0 & 1 & 0 & 0 & 0 \\
\hline Fracture & 0 & 0 & 1 & 0 & 3 & 0 & 1 & 2 & 0 & 0 \\
\hline Reintervention & 3 & 7 & 13 & 13 & NR & NR & NR & NR & 10 & 10 \\
\hline \multicolumn{11}{|l|}{ Surgery and stents } \\
\hline & & \multicolumn{3}{|l|}{ Mehta 2006} & \multicolumn{3}{|c|}{ Jeurnink 2010} & \multicolumn{3}{|l|}{ Fiori 2013} \\
\hline & & Stent & \multicolumn{2}{|c|}{ Surgery } & Stent & \multicolumn{2}{|l|}{ Surgery } & Stent & \multicolumn{2}{|c|}{ Surgery } \\
\hline Total & & 13 & \multicolumn{2}{|c|}{14} & 21 & \multicolumn{2}{|l|}{18} & 9 & \multicolumn{2}{|c|}{9} \\
\hline Technical success & & 10 & \multicolumn{2}{|l|}{13} & 20 & \multicolumn{2}{|l|}{17} & 9 & \multicolumn{2}{|l|}{9} \\
\hline Complications & & 0 & \multicolumn{2}{|l|}{8} & 8 & \multicolumn{2}{|l|}{5} & 6 & \multicolumn{2}{|l|}{6} \\
\hline Reintervention & & NR & \multicolumn{2}{|l|}{ NR } & 7 & 2 & & 4 & \multicolumn{2}{|l|}{1} \\
\hline
\end{tabular}

NR: not reported.

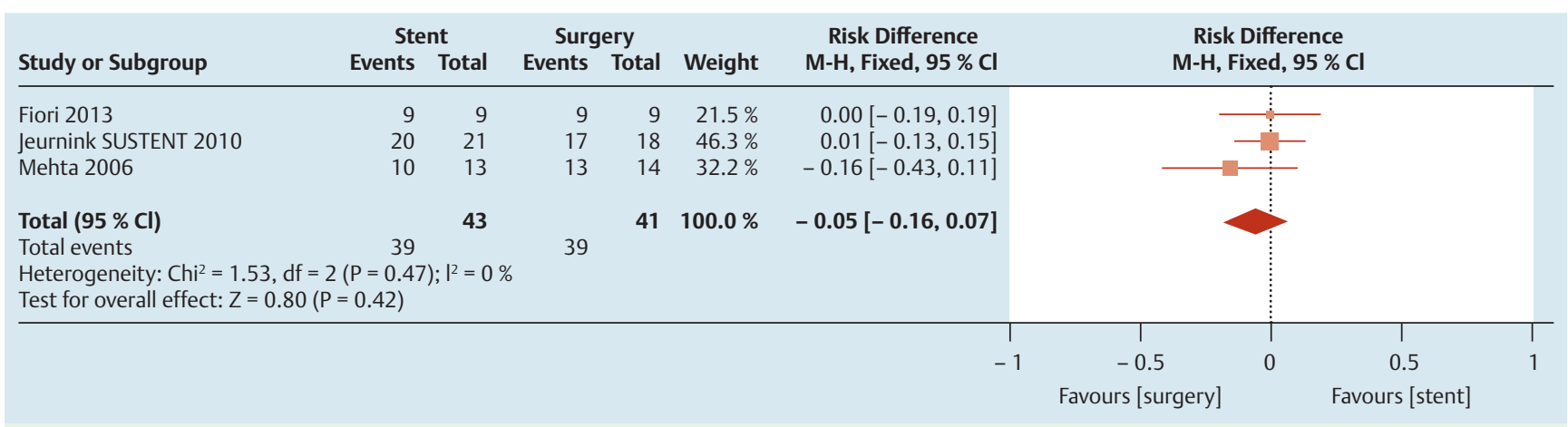

Fig. 2 Forest plot of technical success of surgery and stents, with fixed effect.

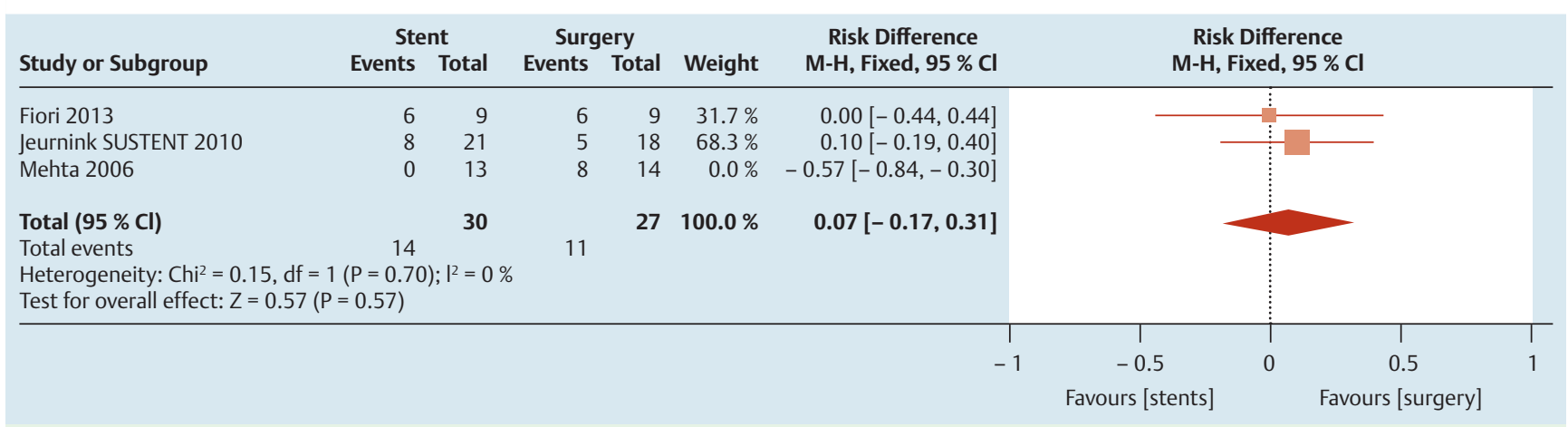

Fig. 3 Forest plot of complications of surgery and stents, with fixed effect, after excluding outlier study.

\section{Complications}

In this analysis, high heterogeneity was found ( $\left.I^{2}: 87 \%\right)$, with one outlier study [16]. After excluding this trial, there is no significant difference between the stents (RD: $-0.08,95 \% \mathrm{CI}:[-0.17,0.00], \mathrm{I}^{2}$ : $6 \%)(\bullet$ Fig. 7).

\section{Obstruction}

Obstruction is one of the key outcomes. Results are favorable to the covered group, with a significant difference (RD:- $0.21,95 \%$ CI $\left.[-0.27,-0.15], I^{2}: 36 \%\right)$ and a NNT of 5 ( $\bullet$ Fig. 8). 


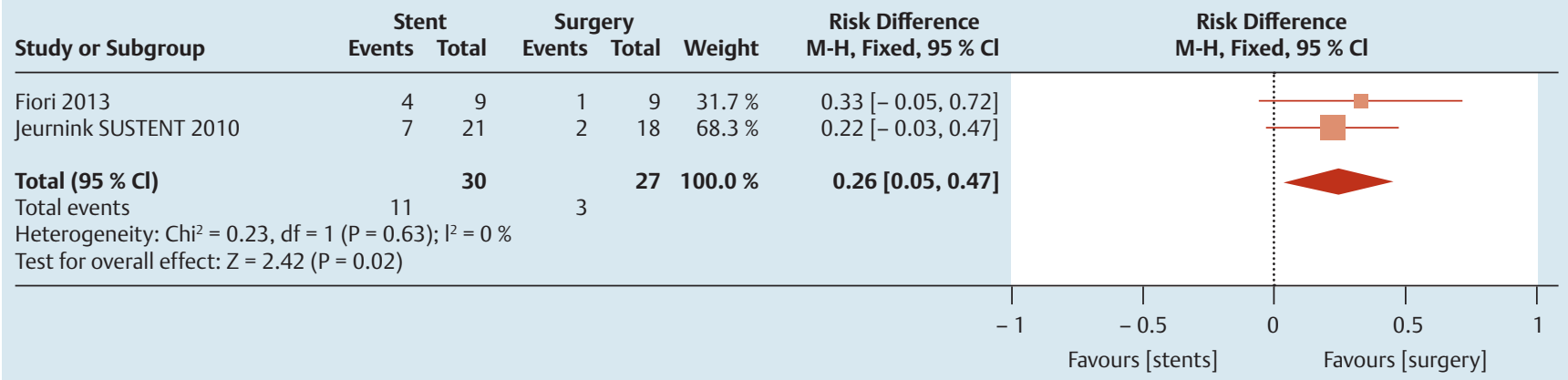

Fig. 4 Forest plot of reinterventions of surgery and stents, with fixed effect.

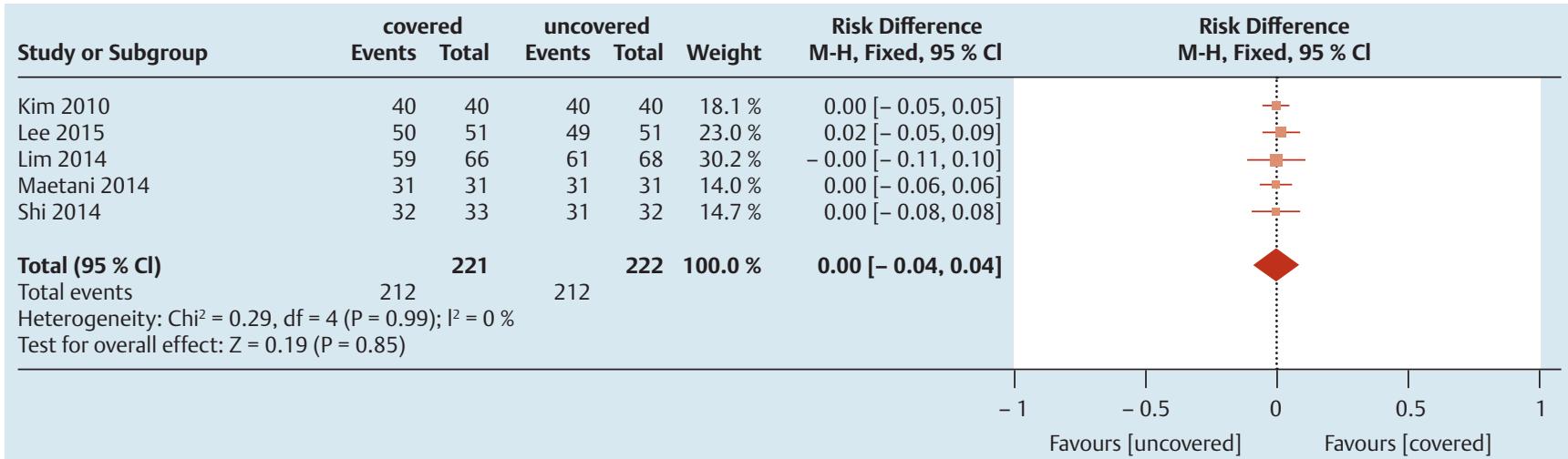

Fig. 5 Forest plot of technical success of covered and uncovered stents, with fixed effect.

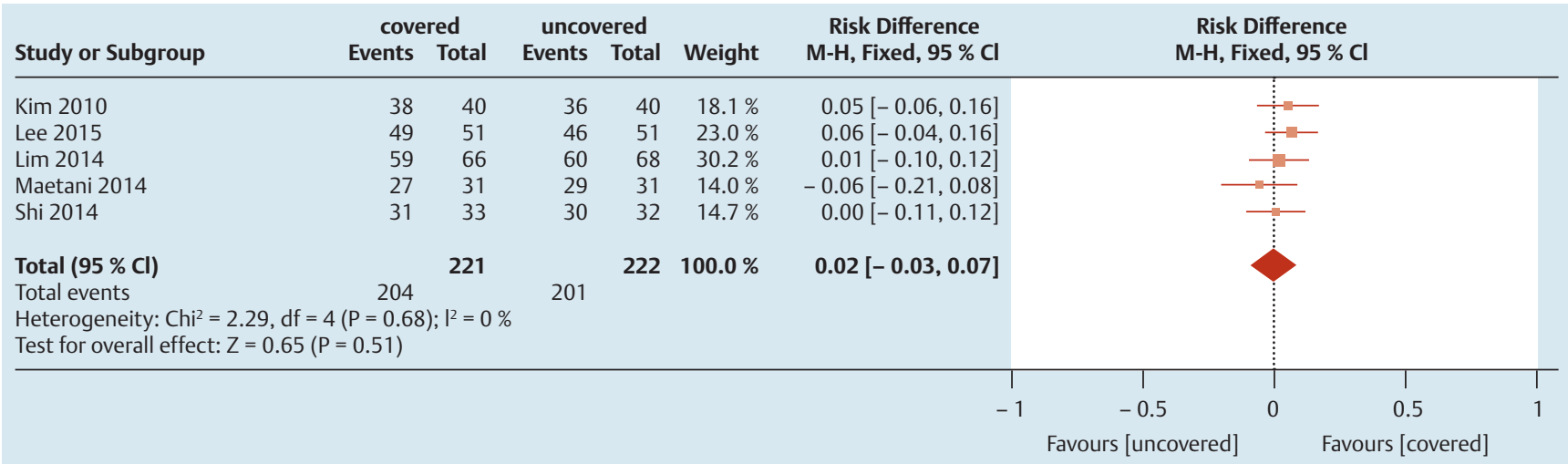

Fig. 6 Forest plot of clinical success of covered and uncovered stents, with fixed effect.

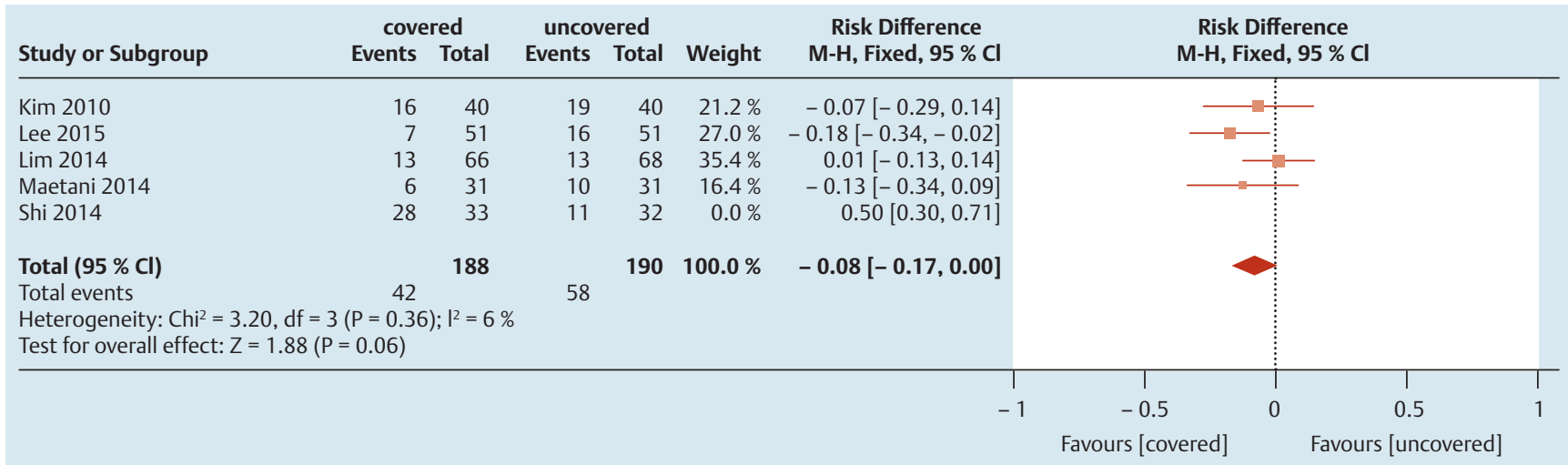

Fig. 7 Forest plot of complications of covered and uncovered stents, with fixed effect, after excluding outlier study. 


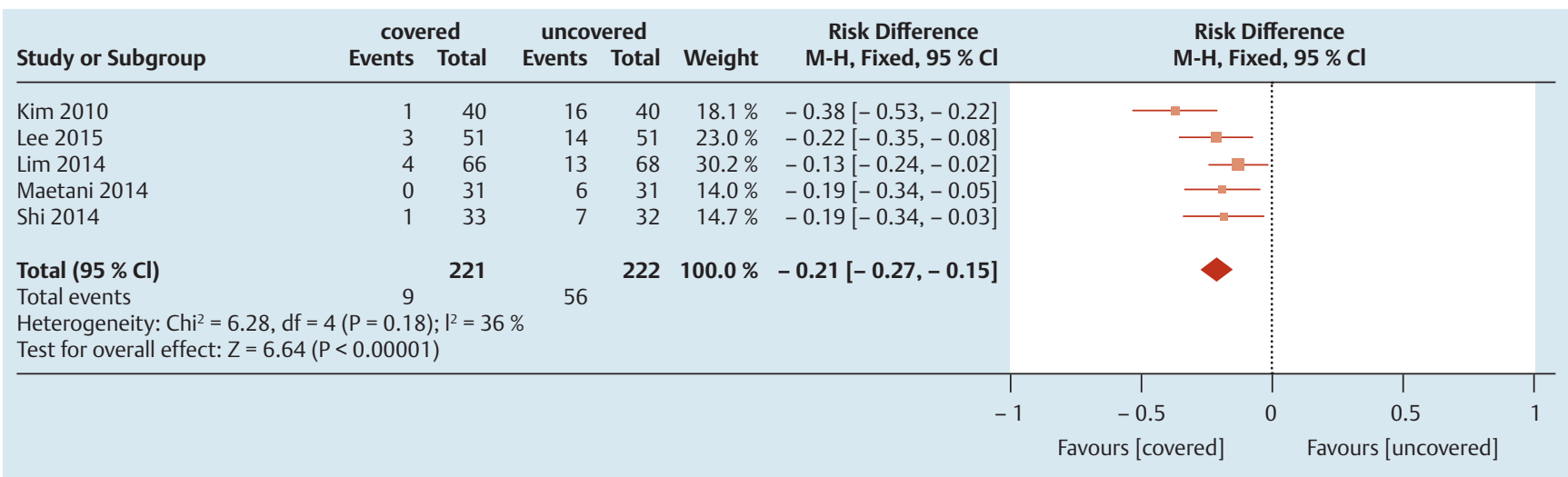

Fig. 8 Forest plot of obstruction of covered and uncovered stents, with fixed effect.

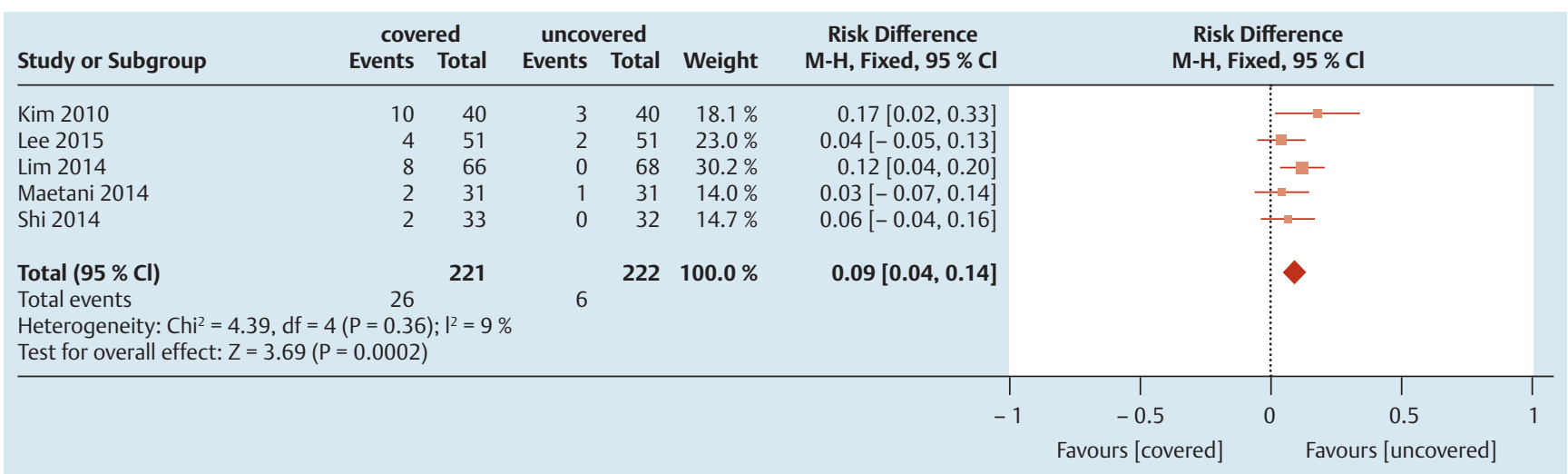

Fig.9 Forest plot of migration of covered and uncovered stents, with fixed effect.

\section{Migration}

Migration is another important outcome that must be considered. The uncovered SEMS showed better results (RD: 0.09, 95\% CI $\left.[0.04,0.14], I^{2}: 9 \%\right)$, with a NNH of 11 . This outcome had low heterogeneity and significant difference ( $\bullet$ Fig.9).

\section{Bleeding}

Analysis is compatible with high heterogeneity ( $\left.\mathrm{I}^{2}: 80 \%\right)$. An outlier study was detected and removed [16], resulting in low heterogeneity and no difference between groups (RD:-0.01, 95\% CI: [-0.03, 0.02], $\mathrm{I}^{2}: 0 \%$ ) ( Fig. 10).

\section{Perforation}

No significant difference was found between the covered and uncovered stents (RD: 0.01, 95\% CI: [-0.01, 0.03], I $\mathrm{I}^{2}$ : 0\% ( Fig. 11).

\section{Fracture}

For this outcome, there is no significant difference between the covered and uncovered stents (RD: 0.01, 95\% CI: [-0.02, 0.04], $\mathrm{I}^{2}$ : $0 \%$ ) (๑ig. 12).

\section{Reintervention}

The covered group showed lower rates of reintervention, with no significant difference. (RD: $-0.03,95 \% \mathrm{CI}$ : [-0.11, 0.06], $\mathrm{I}^{2}$ : $0 \%$ ) (॰ Fig. 13).

\section{Additional analyses}

A subgroup analysis of trials with SEMS, which included only gastric cancer patients, was performed [15, 16,31]. Results of this analysis are very similar to the global analysis (Appendix 2).

\section{Discussion}

Palliation of malignant gastric outlet obstruction can be achieved by surgical and endoscopic techniques [3-7]. Some authors argue that endoscopic treatment is less invasive and should be considered for palliation, since many patients have poor clinical conditions and surgical intervention is associated with higher morbidity and mortality $[8,10]$. Others have a major concern about stent patency, clinical results and the need for reintervention [12]. In our meta-analysis we were able To compare technical success, complications and reinterventions of both techniques. There is a high rate of technical success with both techniques, with few cases of failure and no statistical difference between them. The analysis of complications showed a high heterogeneity between studies. This included an outlier [26], the only one with favorable results for stents. After excluding that one from analysis, there is no significant difference. In the study design, we notice that the follow up time is not until death, so it is possible those results are different because the time of observation was not sufficient to detect complications like obstruction, migration and stent fracture. Early and late publications of one included study showed different results; with a higher number of complications when considering follow up until death [11,27]. Besides, analysis can be influenced by other biases because the selected studies used different kinds of stents (covered and uncovered) and included open and laparoscopy gastrojejunostomy. Considering the need of reintervention, results are favorable to the surgery group, with a significant difference.

When comparing stents and surgery, we also noticed that stents had different results for hospital stay in all included trials [12,26, 


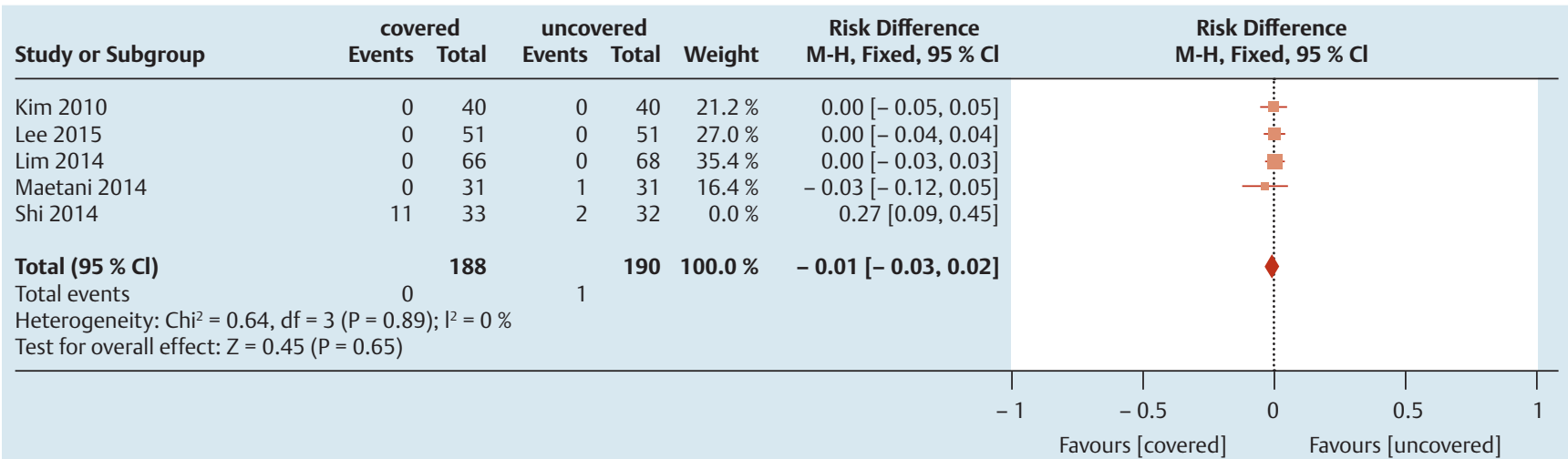

Fig. 10 Forest plot of bleeding of covered and uncovered stents, with fixed effect, after excluding outlier study.

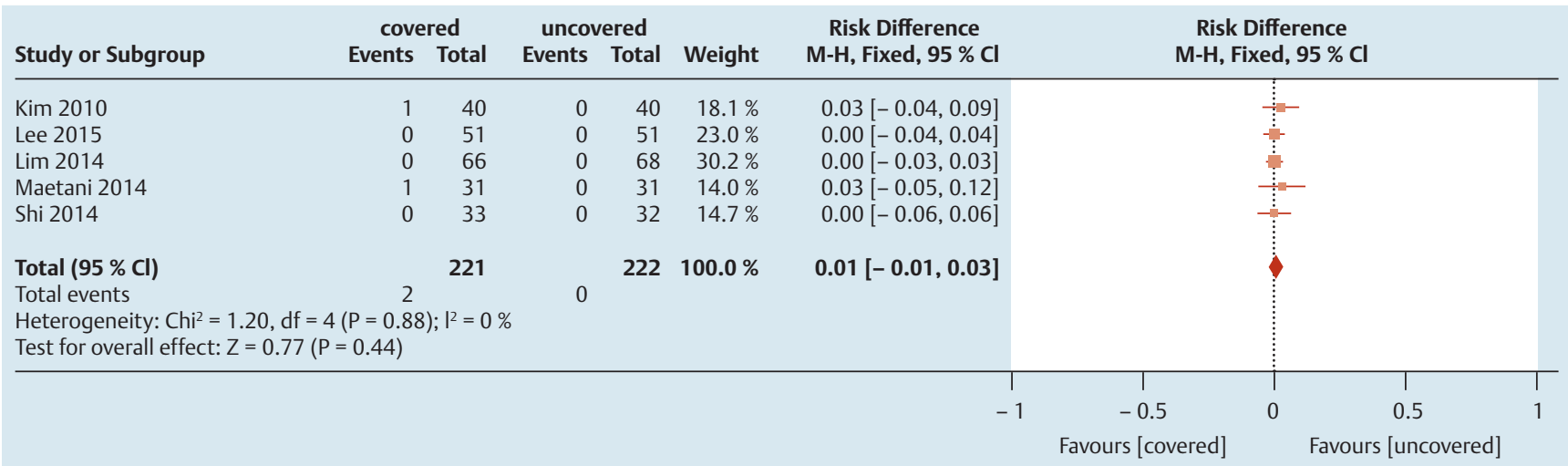

Fig. 11 Forest plot of perforation of covered and uncovered stents, with fixed effect.

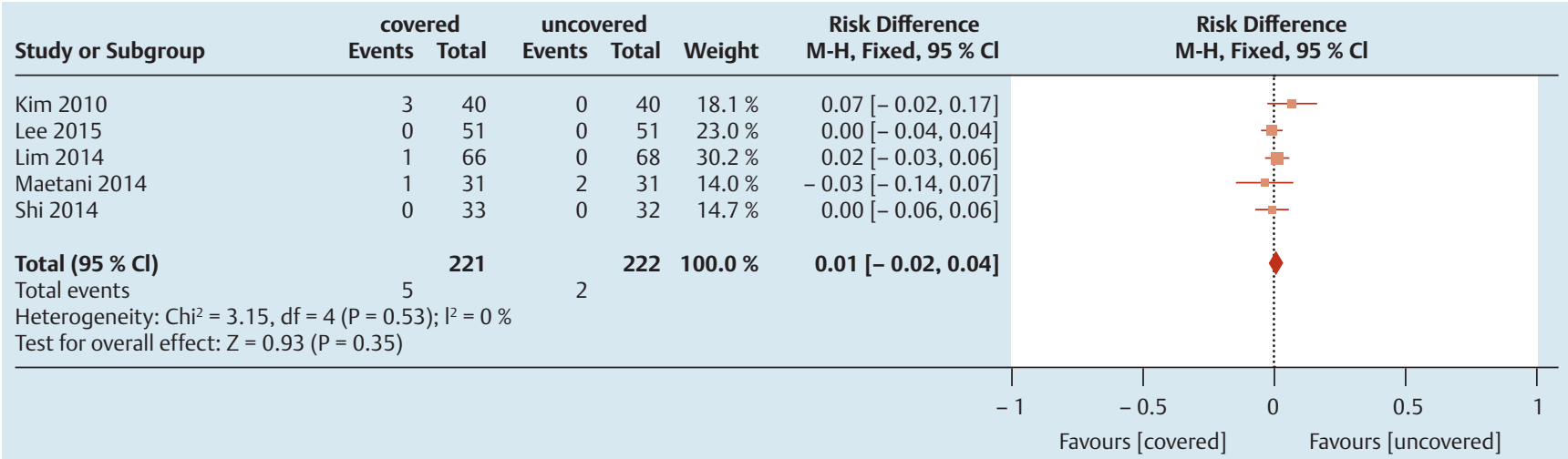

Fig. 12 Forest plot of fracture of covered and uncovered stents, with fixed effect.

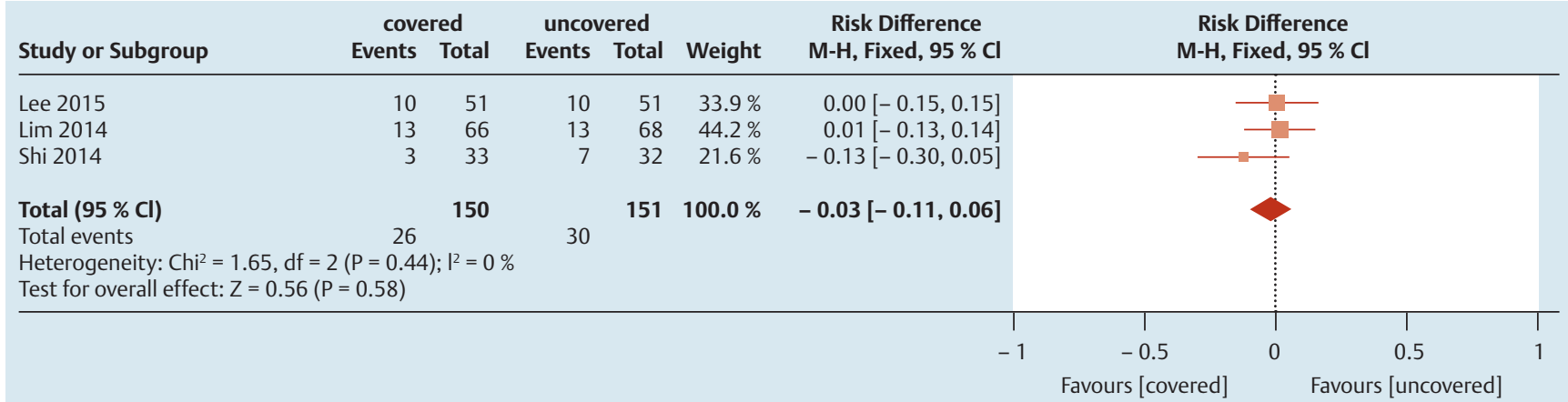

Fig. 13 Forest plot of reintervention of covered and uncovered stents, with fixed effect. 
27]. The series reported 4.8 to 7 median days for stents and 11.4 to 15 for surgery. These data were not included for meta-analysis because standard deviation was not published.

Endoscopic therapy with stents is a valid method for palliation of malignant gastric outlet obstruction $[10,13]$. With new stents available, we question which is the best to use. In this meta-analysis, there are high technical and clinical success rates for SEMS, of around $95 \%$ and $90 \%$, respectively. There is no significant difference between groups.

Complications are one of the key elements for analysis. Studies reported perforation, bleeding, abdominal pain, obstruction and migration of the SEMS [13,32-35]. High heterogeneity was observed in the overall complications analysis. This was attributed to 1 trial [16], which is the only one favorable to the uncovered group. After a sensitivity analysis, we excluded this study for this outcome, with no significant difference. When assessing the individual characteristics of this trial, we observe that this is the only one with power for this outcome (98.70\%) and the stent used is a tailored one. This author reported complications in $84.35 \%$ of covered and $34.38 \%$ of the uncovered stents group. The difference is attributed to bleeding and abdominal pain, with $33.3 \%$ and $39.39 \%$ of all patients submitted to covered SEMS, respectively. When exploring the possible complications, obstruction and migration must be considered. The studies have a major concern with these outcomes. Uncovered stents had more cases of obstruction and covered stents had higher migration rates, both with significant difference. For every five covered SEMS used, there is an obstruction outcome benefit for one patient. However, for every 11 covered stents used, one patient is harmed, as a result of migration. Looking at the trials individually, the worst reported results showed $25 \%$ of migration of covered stents and $40 \%$ obstruction of the uncovered SEMS [31].

A higher number of stent fractures was noted in the covered group, but with a low number of cases $(2.26 \%$ in the covered and $0.90 \%$ in the uncovered group), with no significant difference. The rate of reintervention was lower in the covered group, but with a small and non-significant difference. Despite that, the risk of this outcome is around $20 \%$ for covered or uncovered SEMS, which means that 1 in 5 cases will need some kind of reintervention.

Unfortunately, patency rate could not be included in this systematic review. There is a lack of uniformity of this data, with different forms to express those results and standard deviation is missing in some reports. The patency rate, expressed in median was 68 days for covered and 88 for uncovered ones in the trial with the worst result [17]. Another RCT showed 95 days for covered and 92 for uncovered SEMS [30]. One study expresses the total patency for patients, favoring covered SEMS, with $14 / 31$ (45.2\%) patent cases for covered and 13/36 (36.1\%) for uncovered [31]; but considering intention to treat analysis, results are similar. Another way to interpret the patency rate is correlating with periods. One author showed an 8-week patency rate of $37 / 51$ (72.5\%) for covered and 32/51 (62.7\%) for uncovered SEMS and 16 week patency rates of $35 / 51(68.6 \%)$ and $21 / 51$ (41.2\%), respectively [15]. Results are seemingly not so favorable for either stents in the long term.

Subgroup analysis of trials that included only gastric cancer patients produced very similar results to the complete meta-analysis, with no significant difference for technical and clinical success, complications, fracture and migration. There was a high heterogeneity in complications, which was interpreted with a random effects model. Similar results were noticed, favoring covered
SEMS in the obstruction outcome and uncovered SEMS in the migration outcome.

\section{Strengths and limitations}

The strengths of this systematic review are the inclusion of only randomized clinical trials and global analysis of the palliative treatment of malignant gastric outlet obstruction, which included the surgery, and covered and uncovered stents. One limitation is the selection bias for some studies. In the stents group, 3 studies included only patients with gastric cancer. In addition, the comparison between covered and uncovered stents used different kinds of stents, like tailored ones and those with an anti-migration design. A subgroup analysis was done to verify this bias. Some outcomes could not be compared, including Gastric Outlet Obstruction Scoring System (GOOSS) post procedure, GOOSS change, patency rates and mean survival because lack of uniformity and standard deviation. It would be interesting to have more randomized trials comparing surgery and SEMS, since there are few studies, with low number of patients, resulting in a limited analysis.

\section{Conclusion}

\section{$\nabla$}

When comparing stent types, there is a higher migration rate in the covered stent therapy compared to uncovered stents in the palliation of malignant GOO. Nevertheless, covered SEMS had lower obstruction rates. There is no significant difference in technical success, clinical success, complications, bleeding, perforation, stent fracture and need for reintervention. Surgery is associated with lower rates of reintervention than stents. Both methods present high technical success. Although endoscopic and surgical complications are different, the frequency of this outcome is similar.

\section{Competing interests: None}

\section{References}

1 Jaka H, Mchembe MD, Rambau PF et al. Gastric outlet obstruction at Bugando Medical Centre in northwestern Tanzania: a prospective review of 184 cases. BMC Surgery 2013; $13: 41$

2 Cho YK, Shin JH, Oh SY. Significance of palliative gastrojejunostomy for unresectable pancreatic head carcinoma. Hepatogastroenterology 2008; 55: 254-257

3 Adler DG, Baron TH. Endoscopic palliation of malignant gastric outlet obstruction using self-expanding metal stents: experience in $36 \mathrm{pa}-$ tients. Am J Gastroenterol 2002; 97: $72-78$

4 van Halsema EE, Rauws EA, Fockens $P$ et al. Self-expandable metal stents for malignant gastric outlet obstruction: A pooled analysis of prospective literature. World J Gastroenterol 2015; 21: 12468-12481

5 Tringali A, Didden P, Repici $A$ et al. Endoscopic treatment of malignant gastric and duodenal strictures: a prospective, multicenter study. Gastrointest Endosc 2014; 79: 66-75

6 Zhang LP, Tabrizian P, Nguyen $S$ et al. Laparoscopic gastrojejunostomy for the treatment of gastric outlet obstruction. JSLS 2011; 15: 169-173

7 Chopita N, Landoni N, Ross A et al. Malignant gastroenteric obstruction: therapeutic options. Gastrointest Endosc Clin N Am 2007; 17: 533 544

8 Lo NN, Kee SG, Nambiar R. Palliative gastrojejunostomy for advanced carcinoma of the stomach. Ann Acad Med Singapore 1991; 20: 356 358

9 Weaver DW, Wiencek RG, Bouwman DL et al. Gastrojejunostomy: is it helpful for patients with pancreatic cancer? Surgery 1987; 102: 608 613

10 Dormann A, Meisner S, Verin $N$ et al. Self-expanding metal stents for gastroduodenal malignancies: systematic review of their clinical effectiveness. Endoscopy 2004; 36: $543-550$ 
11 Fiori E, Lamazza A, Volpino P et al. Palliative management of malignant antro-pyloric strictures. Gastroenterostomy vs. endoscopic stenting. A randomized prospective trial. Anticancer Res 2004; 24: 269-271

12 Jeurnink SM, Steyerberg EW, van Hooft JE et al. Dutch SUSTENT Study Group. Surgical gastrojejunostomy or endoscopic stent placement for the palliation of malignant gastric outlet obstruction (SUSTENT study): a multicenter randomized trial. Gastrointest Endosc 2010; 71: 490 499

13 Costamagna G, Tringali A, Spicak J et al. Treatment of malignant gastroduodenal obstruction with a nitinol self-expanding metal stent: an international prospective multicentre registry. Dig Liver Dis 2012; 44: $37-43$

14 Park CI, Kim JH, Lee YC et al. What is the ideal stent as initial intervention for malignant gastric outlet obstruction? Dig Liver Dis 2013; 45: $33-37$

15 Lee H, Min BH, Lee JH et al. Covered metallic stents with an anti-migration design vs. uncovered stents for the palliation of malignant gastric outlet obstruction: a multicenter, randomized trial. Am J Gastroenterol 2015; 110: 1440 - 1449

16 Shi D, Ji F, Bao YS et al. A multicenter randomized controlled trial of malignant gastric outlet obstruction: tailored partially covered stents (placed fluoroscopically) versus standard uncovered stents (placed endoscopically). Gastroenterol Res Pract 2014; 2014: 309797

17 Maetani I, Mizumoto Y, Shigoka $\mathrm{H}$ et al. Placement of a triple-layered covered versus uncovered metallic stent for palliation of malignant gastric outlet obstruction: a multicenter randomized trial. Dig Endosc 2014; 26: 192-199

18 Pan YM, Pan J, Guo LK et al. Covered versus uncovered self-expandable metallic stents for palliation of malignant gastric outlet obstruction: a systematic review and meta-analysis. BMC Gastroenterol 2014; 14: 170

19 Yang $Z, W u$ Q Wang $F$ et al. A systematic review and meta-analysis of randomized trials and prospective studies comparing covered and bare self-expandable metal stents for the treatment of malignant obstruction in the digestive tract. Int J Med Sci 2013; 10: 825-835

20 Hosono $S$, Ohtani $H$, Arimoto $Y$ et al. Endoscopic stenting versus surgical gastroenterostomy for palliation of malignant gastroduodenal obstruction: a meta-analysis. J Gastroenterol 2007; 42: 283-290

21 Jeurnink SM, van Eijck CH, Steyerberg EW et al. Stent versus gastrojejunostomy for the palliation of gastric outlet obstruction: a systematic review. BMC Gastroenterol 2007; 7: 18

22 Ly J, O'Grady G, Mittal A et al. A systematic review of methods to palliate malignant gastric outlet obstruction. Surg Endosc 2010; 24: 290 297
23 Nagaraja V, Eslick GD, Cox MR. Endoscopic stenting versus operative gastrojejunostomy for malignant gastric outlet obstruction-a systematic review and meta-analysis of randomized and non-randomized trials. J Gastrointest Oncol 2014; 5: $92-98$

24 Zheng $B$, Wang $X, M a B$ et al. Endoscopic stenting versus gastrojejunostomy for palliation of malignant gastric outlet obstruction. Dig Endosc 2012; $24: 71-78$

25 Liberati A, Altman DG, Tetzlaff J et al. The PRISMA statement for reporting systematic reviews and meta-analyses of studies that evaluate health care interventions: explanation and elaboration. J Clin Epidemiol 2009; 62: e1 - 34

26 Mehta S, Hindmarsh A, Cheong E et al. Prospective randomized trial of laparoscopic gastrojejunostomy versus duodenal stenting for malignant gastric outflow obstruction. Surg Endosc 2006; 20: 239-242

27 Fiori E, Lamazza A, Demasi E et al. Endoscopic stenting for gastric outlet obstruction in patients with unresectable antro pyloric cancer. Systematic review of the literature and final results of a prospective study. The point of view of a surgical group. Am J Surg 2013; 206: 210-217

28 Jeurnink SM, Polinder S, Steyerberg EW et al. Cost comparison of gastrojejunostomy versus duodenal stent placement for malignant gastric outlet obstruction. J Gastroenterol 2010; 45: 537-543

29 Jeurnink SM, Steyerberg EW, Vleggaar FP et al. Dutch SUSTENT Study Group. Predictors of survival in patients with malignant gastric outlet obstruction: a patient-oriented decision approach for palliative treatment. Dig Liver Dis 2011; 43: 548-552

30 Lim SG, Kim JH, Lee KM et al. Conformable covered versus uncovered self-expandable metallic stents for palliation of malignant gastroduodenal obstruction: a randomized prospective study. Dig Liver Dis 2014; 46: 603-608

$31 \mathrm{Kim}$ CG, Choi IJ, Lee JY et al. Covered versus uncovered self-expandable metallic stents for palliation of malignant pyloric obstruction in gastric cancer patients: a randomized, prospective study. Gastrointest Endosc 2010; 72: 25-32

32 Moura EG, Ferreira FC, Cheng $S$ et al. Duodenal stenting for malignant gastric outlet obstruction: prospective study. World J Gastroenterol 2012; 18: 938 -943

33 Keränen I, Udd M, Lepistö A et al. Outcome for self-expandable metal stents in malignant gastroduodenal obstruction: single-center experience with 104 patients. Surg Endosc 2010; 24: 891 - 896

34 Kaw M, Singh S, Gagneja $\mathrm{H}$ et al. Role of self-expandable metal stents in the palliation of malignant duodenal obstruction. Surg Endosc 2003; 17: $646-650$

35 Lopes $C V$, Pesenti C, Bories E et al. Self-expandable metallic stents for palliative treatment of digestive cancer. J Clin Gastroenterol 2008; 42 991-996 


\section{Appendix 1: Full strategy search}

$\nabla$

Databases: Medline, Scopus, Embase, BVS, LILACS, Cochrane and Cinahl.

Searched until 31.10.2015

Medline:

(Gastric Outlet Obstruction OR Gastric outlet obstructions OR Duodenal obstruction) AND (Endoscopy OR Endoscopic OR endoscopic surgical procedure OR endoscopic surgical procedures OR stent OR stents) AND random*

\section{Scopus:}

(Gastric Outlet Obstruction OR Gastric outlet obstructions OR Duodenal obstruction) AND (Endoscopy OR Endoscopic OR endoscopic surgical procedure OR endoscopic surgical procedures OR stent OR stents) AND random*

Field: Title, abstract, subject.

Embase:

gastric AND outlet AND ('obstruction'/exp OR obstruction) OR gastric AND outlet AND obstructions OR duodenal AND ('obstruction'/exp OR obstruction) AND ('endoscopy'/exp OR endoscopy OR endoscopic AND surgical AND ('procedure'/exp OR procedure) OR endoscopic AND surgical AND ('procedures'/exp OR procedures) OR 'stent'/exp OR stent OR 'stents'/exp OR stents) AND random*

BVS:

(Gastric Outlet Obstruction OR Gastric outlet obstructions OR Duodenal obstruction) AND (Endoscopy OR Endoscopic OR endoscopic surgical procedure OR endoscopic surgical procedures $O R$ stent OR stents) AND random*

Field: Title, abstract, subject.

LILACS:

(Gastric Outlet Obstruction OR Gastric outlet obstructions OR Duodenal obstruction) AND (Endoscopy OR Endoscopic OR endoscopic surgical procedure OR endoscopic surgical procedures OR stent OR stents)

Field: words.

\section{Cochrane:}

(Gastric Outlet Obstruction OR Gastric outlet obstructions OR Duodenal obstruction) AND (Endoscopy OR Endoscopic OR endoscopic surgical procedure OR endoscopic surgical procedures OR stent OR stents) AND random*

Field: search all text.

Cinahl:

(Gastric Outlet Obstruction OR Gastric outlet obstructions OR Duodenal obstruction) AND (Endoscopy OR Endoscopic OR endoscopic surgical procedure OR endoscopic surgical procedures OR stent OR stents) AND random*

Field: TX all text

\section{Grey search literature}

Search executed from chapters of endoscopy and gastroenterology books, thesis and references from selected articles and systematic reviews. No complementation to the initial search were added.

Theses:

Searched at university of São Paulo Bank of Thesis (http://www. teses.usp.br/)

Search strategy: obstrução OU gástrica OU gastroduodenal (resumo).

Search strategy: obstruction OR gastric OR gastroduodenal (abstract).

Results: 331 records.

Books:

- Moura EGH, Artifon ELA, Sakai P. Manual do residente de endoscopia digestiva, 1st ed. São Paulo: Manole; 2014.

- Sakai P, Ishioka S, Maluf Filho F. Tratado de endoscopia digestiva diagnóstica e terapêutica, 1st ed. Rio de Janeiro: Atheneu; 2001.

- Cotton PB, Williams CB. Endoscopia gastrointestinal prática; 6th ed. São Paulo: Santos; 2013.

- FBG (Federação Brasileira de Gastroenterologia). Condutas em Gastroenterologia, 1st ed. Rio de Janeiro: Revinter; 2004.

- Averbach M, Safatle-Ribeiro AV, Ferrari Junior AP et al. Endoscopia Digestiva. Diagnóstico e Tratamento (SOBED), 1st ed. Rio de Janeiro: Revinter; 2013.

- Averbach M, Safatle-Ribeiro AV, Ferrari Junior AP et al. Atlas de Endoscopia Digestiva da SOBED, 1st ed. Rio de Janeiro: Revinter; 2011.

- Miszputen SJ. Guia de gastroenterologia, 2nd ed. São Paulo: Manole; 2007. 


\section{Appendix 2: Subgroup analysis of gastric cancer patients}

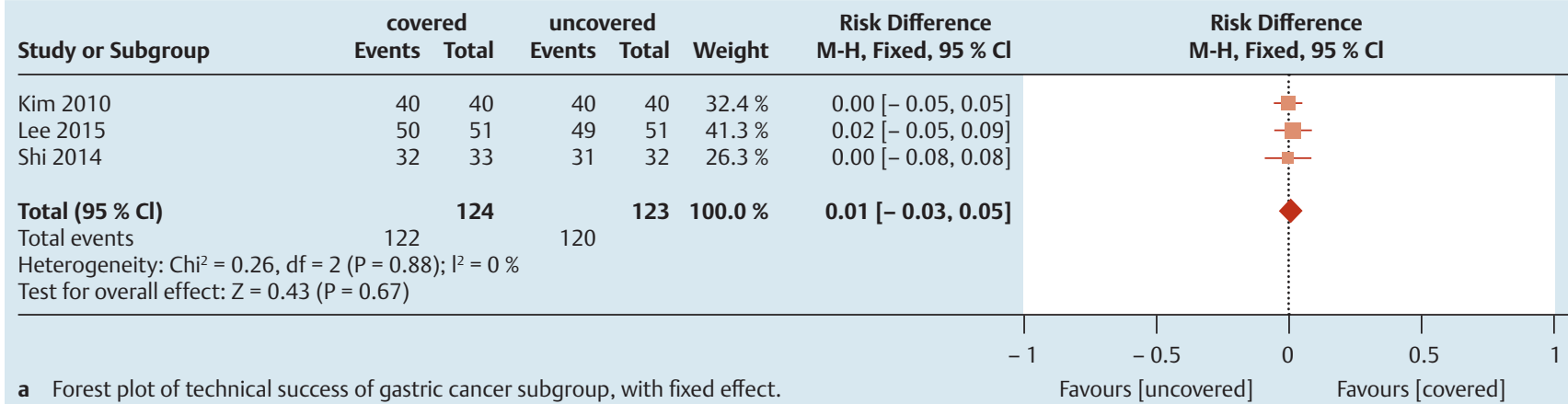

\begin{tabular}{|c|c|c|c|c|c|c|}
\hline \multirow[b]{2}{*}{ Study or Subgroup } & \multicolumn{2}{|c|}{ covered } & \multicolumn{2}{|c|}{ uncovered } & \multirow[b]{2}{*}{ Weight } & \multirow{2}{*}{$\begin{array}{c}\text { Risk Difference } \\
\text { M-H, Fixed, } 95 \% \text { Cl }\end{array}$} \\
\hline & Events & Total & Events & Total & & \\
\hline Kim 2010 & 38 & 40 & 36 & 40 & $32.4 \%$ & $0.05[-0.06,0.16]$ \\
\hline Lee 2015 & 49 & 51 & 46 & 51 & $41.3 \%$ & $0.06[-0.04,0.16]$ \\
\hline Shi 2014 & 31 & 33 & 30 & 32 & $26.3 \%$ & $0.00[-0.11,0.12]$ \\
\hline \multirow{2}{*}{$\begin{array}{l}\text { Total }(95 \% \text { Cl) } \\
\text { Total events }\end{array}$} & & 124 & & 123 & $100.0 \%$ & $0.04[-0.02,0.10]$ \\
\hline & 118 & & 112 & & & \\
\hline \multirow{2}{*}{\multicolumn{7}{|c|}{$\begin{array}{l}\text { Heterogeneity: } \text { Chi }^{2}=0.58, d f=2(P=0.75) ; I^{2}=0 \% \\
\text { Test for overall effect: } Z=1.28(P=0.20)\end{array}$}} \\
\hline & & & & & & \\
\hline \multicolumn{7}{|c|}{ b Forest plot of clinical success of gastric cancer subgroup, with fixed effect. } \\
\hline \multirow[b]{2}{*}{ Study or Subgroup } & \multicolumn{2}{|c|}{ covered } & \multicolumn{2}{|c|}{ uncovered } & \multirow[b]{2}{*}{ Weight } & \multirow{2}{*}{$\begin{array}{c}\text { Risk Difference } \\
\text { M-H, Random, } 95 \% \mathrm{Cl}\end{array}$} \\
\hline & Events & Total & Events & Total & & \\
\hline Kim 2010 & 16 & 40 & 19 & 40 & $32.8 \%$ & $-0.07[-0.29,0.14]$ \\
\hline Lee 2015 & 7 & 51 & 16 & 51 & $34.2 \%$ & $-0.18[-0.34,-0.02]$ \\
\hline Shi 2014 & 28 & 33 & 11 & 32 & $33.1 \%$ & $0.50[0.11,0.71]$ \\
\hline Total (95 \% Cl) & & 124 & & 123 & $100.0 \%$ & $0.08[-0.33,0.50]$ \\
\hline Total events & 51 & & 46 & & & \\
\hline \multicolumn{7}{|c|}{ Heterogeneity: Tau $^{2}=0.12 ;$ Chi $^{2}=27.98, d f=2(P<0.00001) ;\left.\right|^{2}=93 \%$} \\
\hline Test for overall effect & $=0.70)$ & & & & & \\
\hline
\end{tabular}

-

c Forest plot of complications of gastric cancer subgroup, with random effects.

\begin{tabular}{|c|c|c|c|c|c|c|}
\hline \multirow[b]{2}{*}{ Study or Subgroup } & \multicolumn{2}{|c|}{ covered } & \multicolumn{2}{|c|}{ uncovered } & \multirow[b]{2}{*}{ Weight } & \multirow{2}{*}{$\begin{array}{c}\text { Risk Difference } \\
\text { M-H, Fixed, } 95 \% \text { Cl }\end{array}$} \\
\hline & Events & Total & Events & Total & & \\
\hline Kim 2010 & 1 & 40 & 16 & 40 & $32.4 \%$ & $-0.38[-0.53,-0.22]$ \\
\hline Lee 2015 & 3 & 51 & 14 & 51 & $41.3 \%$ & $-0.22[-0.35,-0.08]$ \\
\hline Shi 2014 & 1 & 33 & 7 & 32 & $26.3 \%$ & $-0.19[-0.34,-0.03]$ \\
\hline Total (95 \% Cl) & & 124 & & 123 & $100.0 \%$ & $-0.26[-0.35,-0.17]$ \\
\hline Total events & 5 & & 37 & & & \\
\hline \multicolumn{7}{|c|}{ Heterogeneity: $\mathrm{Chi}^{2}=3.22, \mathrm{df}=2(\mathrm{P}=0.20) ; \mathrm{I}^{2}=38 \%$} \\
\hline Test for overall effect & $<0.0000$ & & & & & \\
\hline
\end{tabular}

\begin{tabular}{l} 
Test for overall effect: $Z=5.85(P<0.00001)$ \\
\hline d Forest plot of obstruction of gastric cancer subgroup, with fixed effect.
\end{tabular}

$\begin{array}{ll}1 & \\ -1 & -0.5 \\ \text { Favours [covered] }\end{array}$

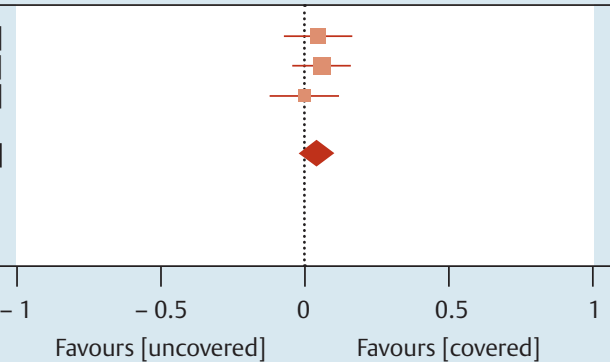

Risk Difference

M-H, Fixed, $95 \%$ Cl

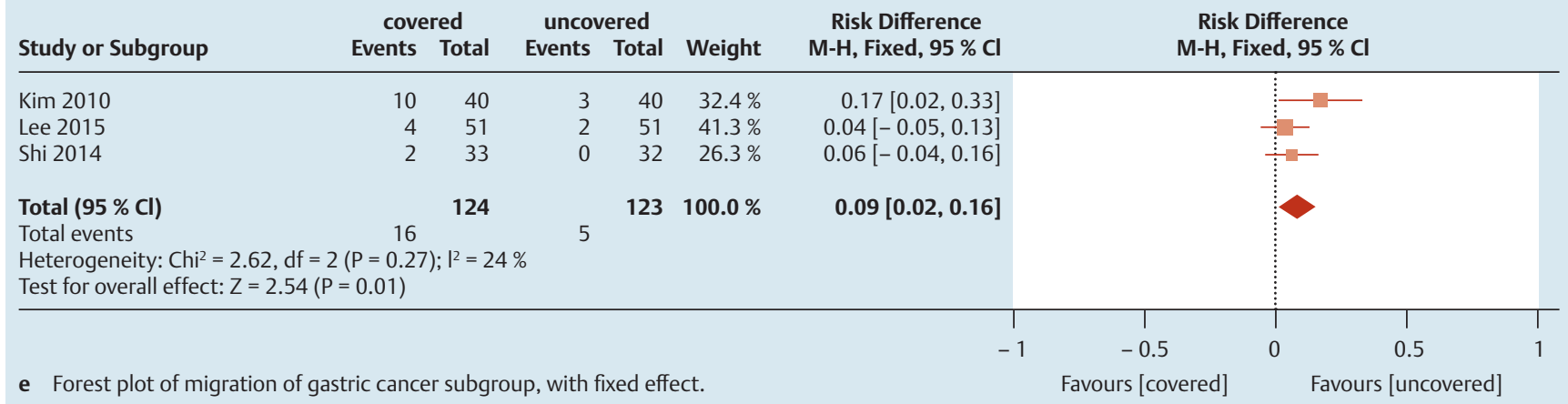




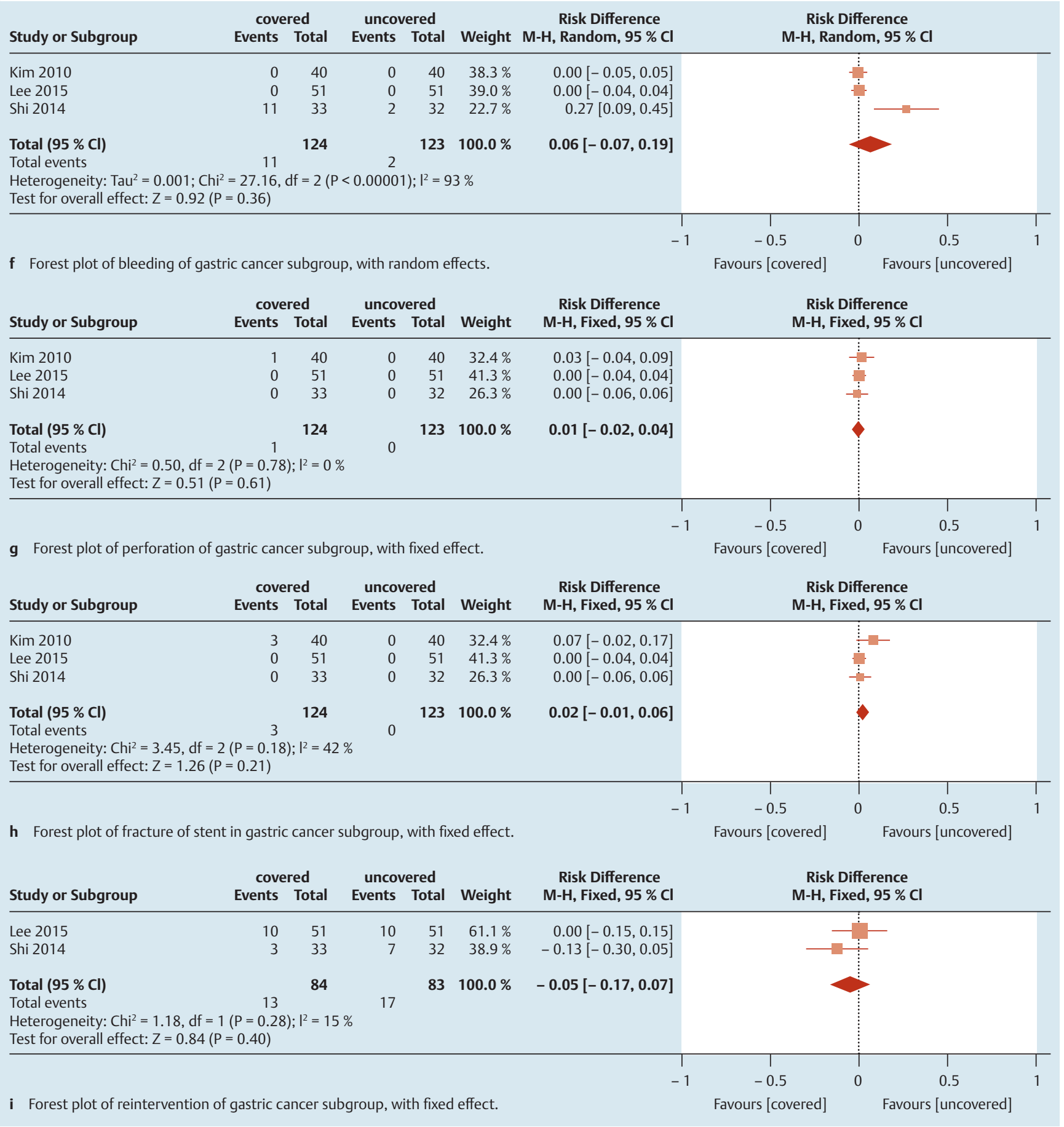

